\section{Caracterização do processo de trabalho das equipes de saúde bucal em municípios de Pernambuco, Brasil, segundo porte populacional: da articulação comunitária à organização do atendimento clínico}

\author{
Characterization of the work process by oral health \\ teams in municipalities in Pernambuco State, Brazil, \\ according to population size: from community links \\ to organization of clinical care
}

1 Centro de Pesquisas Aggeu
Magalhães, Fundação
Oswaldo Cruz, Recife, Brasil.
Correspondência
F. C. Pimentel
Centro de Pesquisas Aggeu
Magalhães, Fundação Oswaldo
Cruz.
Av. Professor Moraes Rego s/n,
Campus da UFPE, Cidade
Universitária, Recife, PE
50670-420, Brasil.
nandocastim@yahoo.com.br

Abstract

This study aimed to analyze the work process in oral health teams in Pernambuco State, Brazil, according to population size in the various municipalities (counties), focusing on the relationship with the community and organization of clinical care. Structured questionnaires were applied to 121 oral health teams in 29 cities. The chi-square test was used to verify the association between the actions and the municipalities' population size. In the integration of the oral health teams with the community, there was a greater focus on schools (81.2\%), while integration with churches $(p=0.000)$ and daycare centers $(p=0.000)$ was associated with larger municipalities. Organization of clinical care showed attention to priority groups (82.3\%), while dental visits were still largely scheduled by spontaneous demand, especially in the smaller municipalities. It is thus necessary to invest in continuing education to adjust the work processes by the team and reduce the differences between practices in the various municipalities in Pernambuco State.

Public Health Dentistry; Dental Health Services; Family Health Program; Oral Health
Fernando Castim Pimentel 1 Paulette Cavalcanti de Albuquerque 1 Petrônio José de Lima Martelli 1 Wayner Vieira de Souza 1 Raquel Moura Lins Acioli 1

\section{Introdução}

A inserção da Saúde Bucal no Programa Saúde da Família (PSF) criou a possibilidade de se instituir um novo paradigma de planejamento e programação da atenção básica e representou, segundo Zanetti 1, a mais importante iniciativa de assistência pública, expandindo e reorganizando as atividades de saúde bucal de acordo com os princípios e diretrizes do Sistema Único de Saúde (SUS). Objetivou-se com isso uma mudança no modelo odontológico anterior, centrado no alívio da dor e no trabalho dentro das quatro paredes do consultório, que inviabilizavam a efetivação do acesso, para um modelo de vigilância à saúde que enfatiza a promoção da saúde e a prevenção das doenças.

Entretanto, para concretizar a inserção da Odontologia no PSF, é preciso entendê-lo como uma estratégia para consolidação do SUS, respeitando seus princípios de participação popular, integralidade, equidade, universalidade, hierarquização e regionalização ${ }^{2}$, o que permite ampliar o acesso da população às ações de saúde bucal, assim como melhorar a qualidade dos serviços oferecidos.

Percebe-se, então, que o número de equipes de saúde bucal (ESB) vem crescendo ao longo dos anos no Brasil 3. Em Pernambuco, no ano de 2001, o estado contava com 132 ESB distribuídas em 39 municípios e, em 2009, o número de equipes perfazia um total de 1.296, distribu- 
ídas em 180 municípios, o que representou um aumento de $881,8 \%$ no quantitativo de equipes implantadas 4 .

Com isso, o Ministério da Saúde pretende que a saúde bucal, na Estratégia Saúde da Família (ESF), expresse os princípios e as diretrizes do SUS e amplie o acesso da população às suas ações de saúde, com a devida integração a uma rede de serviços, estabelecendo-se um sistema de referência e contrarreferência que garanta resolutividade e possibilite o acompanhamento dos pacientes 5 .

Para que essas mudanças possam realmente substituir as práticas tradicionais exercidas nas Unidades Básicas de Saúde (UBS), o Ministério da Saúde passou a definir as características que espera ver consolidadas com a implantação das ESB na ESF, dentre elas: adscrição da população; integralidade da assistência; articulação da referência e contrarreferência aos serviços de média e alta complexidade; definição da família como núcleo central de abordagem; humanização do atendimento; abordagem multiprofissional; estímulo às ações de promoção de saúde, à articulação intersetorial, à participação e ao controle social; educação permanente dos profissionais; além do acompanhamento e avaliação permanente das ações realizadas 6 .

A importância do trabalho em equipe na ESF é ressaltada, principalmente, pelo aspecto de integralidade nos cuidados de saúde, sendo considerada de grande importância para a consolidação de um novo modelo de atenção à saúde no Brasil 7. O planejamento das ações também representa um diferencial nessa estratégia, havendo a necessidade de organizar a Atenção em Saúde Bucal, por meio de ferramentas do planejamento estratégico, que utilize critérios para priorização dos casos 8 , possibilitando, assim, a organização do atendimento clínico.

Apesar do grande esforço para efetivação dessa nova forma de cuidado, as equipes de saúde bucal ainda encontram dificuldades para a realização das práticas pertinentes à estratégia, como visitas domiciliares pelo dentista, ações de prevenção e promoção à saúde, bem como reuniões com a comunidade de abrangência ${ }^{9}$. Supõe-se que muitas dessas dificuldades estejam relacionadas ao porte populacional dos municípios, pois os de grande porte, com maior poder econômico, teriam uma melhor estrutura de serviços disponível para o desenvolvimento das ações de saúde, quando comparados aos municípios de médio e pequeno porte.

Assim, este trabalho teve como objetivo analisar o processo de trabalho das equipes de saúde bucal no Estado de Pernambuco, Brasil, segundo porte populacional, enfocando a articulação junto à comunidade e a organização do atendimento clínico.

\section{Metodologia}

Trata-se de uma pesquisa avaliativa caracterizada como estudo de corte transversal do tipo amostral, representativo para o Estado de Pernambuco.

O Estado de Pernambuco está dividido em 185 municípios, que, segundo estimativas intercensitárias do Instituto Brasileiro de Geografia e Estatística (IBGE) para 2008, tem uma população de 8.734 .194 habitantes, dos quais $89,13 \%$ são cobertos pela ESF ou pelo Programa de Agentes Comunitários de Saúde (PACS), os quais apresentaram uma rápida expansão nos últimos dez anos, atingindo todos os municípios do estado e o distrito de Fernando de Noronha. Segundo o Cadastro Nacional de Estabelecimentos de Saúde (CNES), do Ministério da Saúde, o Estado de Pernambuco possuía, em agosto de 2008, 1.815 equipes de saúde da família e 1.181 equipes de saúde bucal 4 .

Dos 185 municípios, apenas 10 possuem mais de 100 mil habitantes e têm tido acesso a recursos próprios na estruturação da ESF por meio do Projeto de Expansão e Consolidação da Saúde da Família (PROESF), podendo desenvolver mecanismos de capacitação dos profissionais de forma autônoma, de melhoria das condições de trabalho das unidades, compra de equipamentos etc. Os demais municípios contam apenas com os recursos repassados para o fundo municipal de saúde, a título do Piso da Atenção Básica (PAB) ou dos incentivos para equipes de saúde da família, agentes comunitários de saúde ou equipes de saúde bucal. Para este estudo, considerou-se município de pequeno porte aquele com menos de 20 mil habitantes; médio porte o que possuir entre 20 mil e 100 mil habitantes; e o município com mais de 100 mil habitantes foi considerado de grande porte.

Optou-se por uma amostra aleatória estratificada, significativa para todo o Estado de Pernambuco, calculada a partir de um nível de 95\% de confiança, margem de erro de $5 \%$ e uma prevalência de $91 \%$, com base em um estudo realizado por Baldani et al. 9 , cujo resultado encontrou que 91\% das ESB realizam o planejamento das ações. Dada a existência de 1.181 (em agosto de 2008) equipes de saúde bucal no estado, a amostra foi calculada em 114 equipes, usando-se o programa Epi Info versão 6.04 (Centers for Disease Control and Prevention, Atlanta, Estados Unidos).

A distribuição dos questionários a serem aplicados nos municípios foi baseada na proporção 
de ESB existentes em Pernambuco, distribuída por porte populacional. Assim, deveriam ser realizados 34 questionários nos municípios de grande porte, 57 nos municípios de médio e 30 naqueles de pequeno porte (Tabela 1). Entretanto, ao final da coleta dos dados, observou-se uma diferença entre os porcentuais de questionários que deveriam ser aplicados e aqueles realmente aplicados. Tal diferença está ligada à vinculação da aplicação dos questionários das ESB aos questionários do projeto principal da ESF: em muitas unidades de Saúde da Família visitadas não havia ESB, ou, em alguns municípios, existia um número superior de ESB àquele previamente calculado. Com isso, a fim de corrigir esse porcentual, foi calculado um fator de ponderação, dividindo-se, para cada porte populacional, a proporção de questionários calculados pela proporção de questionários aplicados. Esse fator é de grande importância na apresentação dos resultados, pois cada item analisado do questionário foi ponderado por porte e o seu resultado expresso com a devida correção.

Os dados primários foram coletados entre julho de 2009 e março de 2010, por meio da aplicação de um questionário estruturado às equipes de saúde bucal participantes da amostra. O questionário foi aplicado pelos integrantes da pesquisa que passaram por treinamento antes da coleta dos dados, a fim de reduzir um possível viés do entrevistador.

No questionário foram analisadas variáveis como:

1. A integração da equipe de saúde bucal com os demais profissionais da ESF, por meio de atividades como reuniões, análise em conjunto da situação de saúde da população, planejamento integrado das ações da equipe para participação das ações.

2. Participação nos grupos educativos. As atividades desenvolvidas por esses grupos têm o objetivo de buscar adesão do paciente ao tratamen- to, à prevenção de risco e ao controle de doenças. As ações pretendidas para esses grupos circulam pelo campo da educação em saúde, com uma forte base na informação e esclarecimento da doença ou o agravo 10.

3. O diagnóstico da área, que consiste no levantamento das condições socioeconômicas e sanitárias da população adscrita, na identificação de equipamentos sociais, no levantamento epidemiológico e outras informações importantes para o desenvolvimento das ações de saúde da equipe. O levantamento epidemiológico por sua vez, consiste na coleta e análise de dados sobre as doenças bucais (cárie, doença periodontal e outras) na comunidade, bem como na análise de indicadores de impacto das ações adotadas. Na pergunta referente a esta variável, orientouse que o entrevistado considerasse a realização do diagnóstico se o mesmo foi há, pelo menos, um ano.

4. A organização do atendimento foi dividida e repassada aos entrevistados, podendo ser: por faixa etária (criança, adolescente, adulto, idoso), por grupos prioritários (gestantes, hipertensos, diabéticos) e por risco (mães analfabetas, baixo nível socioeconômico). Além dessas formas de organização, também foram incluídas a demanda programada, estabelecida por meio do cadastramento familiar ou pelo levantamento epidemiológico; marcação por microárea, realizada pelos agentes comunitários de saúde (ACS); agenda aberta, em que todos os usuários que comparecem à unidade, a qualquer horário do dia, e são agendados para atendimento, quando houver vaga; e demanda espontânea, quando os usuários comparecem ao serviço à procura de atendimento, diariamente ou semanalmente, e competem por um número de fichas ou vagas para atendimento no período. Nessa última forma de organização, são formadas filas, por vezes, de madrugada, de usuários em busca da consulta odontológica. O Ministério da Saúde, em 2004 11,

Tabela 1

Distribuição das equipes de saúde bucal (ESB) em Pernambuco, Brasil (agosto de 2008), questionários aplicados e fator de correção.

\begin{tabular}{|c|c|c|c|c|c|}
\hline \multirow[t]{2}{*}{ Porte populacional dos municípios } & \multicolumn{2}{|c|}{ ESB } & \multicolumn{2}{|c|}{ Questionários aplicados } & \multirow{2}{*}{$\begin{array}{c}\text { Fator de } \\
\text { ponderação }\end{array}$} \\
\hline & $\mathrm{n}$ & $\%$ & $\mathbf{n}$ & $\%$ & \\
\hline Grande & 335 & 28,4 & 57 & 47,1 & 0,602 \\
\hline Médio & 554 & 46,9 & 37 & 30,6 & 1,534 \\
\hline Pequeno & 292 & 24,7 & 27 & 22,3 & 1,108 \\
\hline Total & 1.181 & 100,0 & 121 & 100,0 & \\
\hline
\end{tabular}


incentivou o desenvolvimento de ações voltadas para as linhas do cuidado, como por exemplo, "da criança, do adolescente, do adulto, do idoso e o desenvolvimento de ações complementares voltadas para as condições especiais de vida como saúde da mulher, saúde do trabalhador, portadores de necessidades especiais, hipertensos, diabéticos, dentre outras".

5. A articulação das equipes com a comunidade, através reuniões para debater temas sobre cidadania, assistência prestada e funcionamento do SUS, reuniões com conselhos de saúde e participação da comunidade no processo de planejamento do trabalho das ESB.

Foi selecionado um informante-chave (cirurgião-dentista - CD, auxiliar de saúde bucal-ASB, ou técnico em saúde bucal - TSB) de cada ESB para responder ao questionário, devido, em muitos casos, à dificuldade de paralisar o turno de atendimento da equipe. Entretanto, foi necessário que esse informante apresentasse alguns critérios como ser integrante da ESB há pelo menos um ano e conhecer as atividades desenvolvidas pela equipe, garantindo, assim, uma melhor qualidade das informações.

Foi aplicado um questionário piloto, inicialmente em uma ESB selecionada intencionalmente, pela facilidade de contato e disponibilidade das equipes em respondê-lo. O teste piloto é de grande importância na detecção de inconsistências e ajustes no instrumento de coleta (questionário). A adaptação, a correção e a identificação de alguns aspectos relativos à estrutura oferecida às equipes e o desempenho de suas atividades favoreceram uma avaliação mais fidedigna e completa do modelo de atenção desenvolvido pelas ESB na ESF.

Ao longo de 6 meses (julho a dezembro de 2009), foram visitados 22 municípios, perfazendo um total de 89 equipes de saúde bucal entrevistadas in loco. Sem recursos financeiros, o que impossibilitou a viagem para novos municípios, as informações passaram a ser coletadas via contato telefônico com os coordenadores de saúde bucal dos municípios, os quais passaram os telefones das ESB para o contato direto com elas. Houve uma maior dificuldade para entrar em contato com alguns municípios e, quando foi possível, muitas equipes não tinham disponibilidade para responder ao questionário. De fevereiro a março de 2010, foi realizada a aplicação de 32 questionários em 7 municípios do Estado de Pernambuco, por meio de contato telefônico. É importante ressaltar que, após a realização dessas entrevistas, foi enviado ao município um envelope lacrado com os Termos de Consentimento Livre e Esclarecido para a assinatura dos profissionais que participaram, além de um en- velope selado para o retorno destes termos sem custo para o município.

Para realização da análise dos dados, organizou-se uma base de dados no programa Epi Info, versão 6.04, elaborada após a digitação dos questionários, em que obtivemos informações de cada ESB.

Vale ressaltar que, para cada item analisado, foi realizada a estratificação por porte populacional e, aos resultados referentes a Pernambuco, foi aplicado o fator de ponderação, referido anteriormente, o qual corrige a influência das proporções dos questionários calculados/aplicados entre os portes municipais nas respostas analisadas. Os resultados foram expressos em proporções no formato de tabelas.

A análise dos questionários também permitiu verificar se existe diferença estatística significante entre as ações realizadas pelas equipes de saúde bucal e o porte populacional dos municípios. Para isso, utilizou-se o teste qui-quadrado para comparar as proporções entre esses três estratos, usando-se o programa Epi Info 6.04.

Este trabalho foi parte integrante do projeto de pesquisa Atenção Primária à Saúde no Estado de Pernambuco: Avaliação do Período 19972006 Segundo Modelo de Gestão dos Municípios, financiado pelo Conselho Nacional de Desenvolvimento Científico e Tecnológico (CNPq). O mesmo cumpre os princípios éticos para pesquisa envolvendo seres humanos, contidos na Declaração de Helsinki (2000), e se orienta conforme os parâmetros bioéticos da Resolução $n^{\circ}$. 196/96 (Comissão Nacional de Ética em Pesquisa), tendo sido aprovado pelo Comitê de Ética em Pesquisa do Centro de Pesquisas Aggeu Magalhães, Fundação Oswaldo Cruz (CPqAM/Fiocruz, $n^{\circ}$. 129/2008) em 4 de dezembro de 2008. Os entrevistados foram esclarecidos sobre os objetivos do trabalho, tendo sua identidade preservada e convidados a assinar um Termo de Consentimento Livre e Esclarecido, garantindo-se, assim, o sigilo da autoria das informações.

\section{Resultados}

\section{A articulação entre as ESB e a comunidade}

Sobre a participação das equipes nas atividades de grupos educacionais, percebe-se uma maior integração com os grupos de gestantes $(68,4 \%)$, idosos $(62,1 \%)$ e hipertensos/diabéticos (61,3\%). Sua menor participação nos grupos de adolescentes, portadores de deficiência e outros talvez seja consequência da dificuldade de formar e/ou inexistência destes grupos nas unidades, enquanto os primeiros são mais frequentes (Tabela 2). 
Na integração das ESB com instituições e organizações da comunidade (Tabela 2), nota-se seu foco voltado para a população infantil nas escolas $(81,2 \%)$. As demais instituições apresentaram um porcentual baixo de articulação com as ESB, muitas vezes devido à inexistência desses grupos na área de abrangência. Considerando as diferenças por porte populacional, observou-se um maior envolvimento das equipes dos municípios de médio porte com associações ( $\mathrm{p}=0,038)$, e das equipes dos municípios de grande porte com igrejas $(p=0,000)$ e creches $(p=0,000)$.

Sobre os processos de participação popular no Saúde da Família, constatou-se que apenas 24,0\% das ESB reúnem-se com a comunidade periodicamente para debater temas sobre cidadania, assistência, funcionamento do SUS e outros; $46,2 \%$ das equipes possuem um integrante que participa das reuniões com o Conselho de Saúde; e em 19,3\% existem a participação de representantes de movimentos sociais e usuários no processo de planejamento do trabalho da equipe (Tabela 2).

\section{A organização do processo de trabalho}

Com base na Tabela 3, observou-se que grande parte das ESB de Pernambuco articula-se com os demais membros da equipe de saúde da família, realizando ações como reunião administrativa $(86,4 \%)$, planejamento das ações $(86,0 \%)$, entre outras. Entretanto, talvez a mais importante atividade a ser realizada em conjunto apresentou o menor porcentual dentre as citadas: $68,8 \%$ das equipes realizam a análise da situação de saúde da população em conjunto.

Com relação à utilização do mapa de abrangência da unidade (Tabela 3), pode-se perceber a subutilização dessa ferramenta de trabalho ao se constatar que apenas $37,3 \%$ das equipes utilizaram o mapa do território nos últimos 2 meses. Percebe-se que a maior parte $(32,8 \%)$ se deteve no mapeamento das famílias por território para organização do atendimento clínico.

A atividade de diagnóstico da área, utilizada para a identificação da situação em que se encontra a população adscrita, foi realizada por apenas

Tabela 2

Relação das ações desenvolvidas pelas equipes de saúde bucal (ESB) em articulação com a comunidade por porte populacional. Pernambuco, Brasil, 2009 (respostas múltiplas).

\begin{tabular}{|c|c|c|c|c|c|c|c|c|c|}
\hline \multirow[t]{2}{*}{ Ações } & \multicolumn{2}{|c|}{ Grande porte } & \multicolumn{2}{|c|}{ Médio porte } & \multicolumn{2}{|c|}{ Pequeno porte } & \multicolumn{3}{|c|}{ Total } \\
\hline & $\mathbf{n}$ & $\%$ & $\mathbf{n}$ & $\%$ & $\mathbf{n}$ & $\%$ & $\mathbf{N}$ & $\%$ & Valor de $p$ \\
\hline \multicolumn{10}{|l|}{ Participação da equipe nos grupos educativos } \\
\hline Hipertensos e/ou diabéticos & 40 & 70,2 & 24 & 64,9 & 12 & 44,4 & 74,2 & 61,3 & 0,060 \\
\hline Gestantes & 45 & 78,9 & 24 & 64,9 & 17 & 62,9 & 82,7 & 68,4 & 0,274 \\
\hline Idosos & 40 & 70,2 & 21 & 56,8 & 17 & 62,9 & 75,1 & 62,1 & 0,383 \\
\hline Adolescentes & 30 & 52,6 & 10 & 27,0 & 11 & 40,7 & 45,6 & 37,7 & 0,037 \\
\hline Portadores de deficiência & 12 & 21,0 & 4 & 10,8 & 3 & 11,1 & 16,7 & 13,8 & 0,326 \\
\hline \multicolumn{10}{|l|}{ Articulação da equipe com instituições e outras organizações } \\
\hline ONG & 4 & 7,0 & 0 & 0,0 & 1 & 3,7 & 3,5 & 2,9 & 0,245 \\
\hline Associações & 22 & 38,6 & 22 & 59,5 & 8 & 29,6 & 55,9 & 46,2 & 0,017 \\
\hline Conselhos & 10 & 17,5 & 12 & 32,4 & 6 & 22,2 & 31,1 & 25,7 & 0,320 \\
\hline Igrejas & 26 & 45,6 & 1 & 2,7 & 1 & 3,7 & 18,3 & 15,1 & 0,000 \\
\hline Movimentos sociais & 12 & 21,0 & 3 & 8,1 & 2 & 7,4 & 14,0 & 11,6 & 0,146 \\
\hline Escolas & 50 & 87,7 & 30 & 81,1 & 20 & 74,1 & 98,3 & 81,2 & 0,316 \\
\hline Creches & 44 & 77,2 & 7 & 18,9 & 8 & 29,6 & 46,1 & 38,1 & 0,000 \\
\hline \multicolumn{10}{|l|}{ Participação da comunidade nas atividades da ESB } \\
\hline $\begin{array}{l}\text { A ESB reúne-se com a comunidade periodicamente } \\
\text { para debater temas sobre cidadania, assistência, } \\
\text { funcionamento do SUS, outros }\end{array}$ & 18 & 31,6 & 9 & 24,3 & 4 & 14,8 & 29,1 & 24,0 & 0,203 \\
\hline A ESB participa de reuniões com o Conselho de Saúde & 18 & 31,6 & 20 & 54,0 & 13 & 48,1 & 55,9 & 46,2 & 0,125 \\
\hline $\begin{array}{l}\text { Existe a participação de representantes de movimentos } \\
\text { sociais e usuários no processo de planejamento do } \\
\text { trabalho das ESB }\end{array}$ & 10 & 17,5 & 7 & 18,9 & 6 & 22,2 & 23,4 & 19,3 & 0,842 \\
\hline
\end{tabular}

Nota: os valores de N (total), \% (total) e valor de p da tabela acima já se encontram com o fator de ponderação. 
Forma de organização do processo de trabalho das equipes de saúde bucal (ESB) por porte populacional. Pernambuco, Brasil, 2009 (respostas múltiplas).

\begin{tabular}{|c|c|c|c|c|c|c|c|c|c|}
\hline \multirow[t]{2}{*}{ Ações } & \multicolumn{2}{|c|}{ Grande porte } & \multicolumn{2}{|c|}{ Médio porte } & \multicolumn{2}{|c|}{ Pequeno porte } & \multicolumn{3}{|c|}{ Total } \\
\hline & $\mathbf{n}$ & $\%$ & $\mathrm{n}$ & $\%$ & $\mathrm{n}$ & $\%$ & $\mathbf{N}$ & $\%$ & Valor de $\mathrm{p}$ \\
\hline \multicolumn{10}{|l|}{ Participação de ações integradas com demais membros } \\
\hline \multicolumn{10}{|l|}{ da equipe } \\
\hline Reunião administrativa & 53 & 92,9 & 30 & 81,1 & 24 & 88,9 & 104,5 & 86,4 & 0,175 \\
\hline Análise da situação de saúde da população & 36 & 63,2 & 25 & 67,6 & 21 & 77,8 & 83,3 & 68,8 & 0,439 \\
\hline Planejamento das ações da equipe & 49 & 85,9 & 32 & 86,5 & 23 & 85,2 & 104,1 & 86,0 & 0,988 \\
\hline Participação em campanhas/outros eventos & 47 & 82,5 & 32 & 86,5 & 25 & 92,6 & 105,1 & 86,8 & 0,419 \\
\hline Participação nos grupos educativos & 52 & 91,2 & 29 & 78,4 & 22 & 81,5 & 100,2 & 82,8 & 0,299 \\
\hline Acompanhamento integrado dos casos & 47 & 82,5 & 32 & 86,5 & 23 & 85,2 & 102,9 & 85,0 & 0,863 \\
\hline \multicolumn{10}{|l|}{ Utilizou o mapa da área de abrangência? } \\
\hline Responderam sim & 19 & 33,3 & 14 & 37,8 & 11 & 40,7 & 45,1 & 37,3 & 0,783 \\
\hline \multicolumn{10}{|l|}{ Para que o mapa foi utilizado? } \\
\hline Organizar $\mathrm{o}$ atendimento & 18 & 31,6 & 13 & 35,1 & 8 & 29,6 & 39,6 & 32,8 & 0,887 \\
\hline Demarcar áreas de risco & 10 & 17,5 & 10 & 27,0 & 7 & 25,9 & 29,1 & 24,1 & 0,595 \\
\hline Distribuição das famílias por ACS & 11 & 19,3 & 11 & 29,7 & 9 & 33,3 & 33,5 & 27,7 & 0,487 \\
\hline \multicolumn{10}{|l|}{ É realizado o diagnóstico da área adscrita? } \\
\hline Responderam sim & 36 & 63,2 & 19 & 51,3 & 11 & 40,7 & 63,0 & 52,1 & 0,220 \\
\hline \multicolumn{10}{|l|}{ Para que realizou o diagnóstico da área? } \\
\hline Identificação dos grupos de risco & 26 & 45,6 & 17 & 45,9 & 9 & 33,3 & 51,7 & 42,7 & 0,465 \\
\hline $\begin{array}{l}\text { Identificar escolas, creches, asilos, outras instituições } \\
\text { e grupos comunitários }\end{array}$ & 33 & 57,9 & 19 & 51,3 & 10 & 37,0 & 60,1 & 49,7 & 0,202 \\
\hline $\begin{array}{l}\text { Levantamento das condições socioeconômicas e } \\
\text { sanitárias da população }\end{array}$ & 21 & 36,8 & 17 & 45,9 & 8 & 29,6 & 47,6 & 39,3 & 0,360 \\
\hline Diagnóstico das doenças bucais na comunidade & 27 & 47,4 & 16 & 43,2 & 11 & 40,7 & 52,9 & 43,8 & 0,851 \\
\hline
\end{tabular}

ACS: agentes comunitários de saúde.

Nota: os valores de N (total), \% (total) e valor de p da tabela acima já se encontram com o fator de ponderação.

$52,1 \%$ das equipes entrevistadas. Constatou-se que $49,7 \%$ realizaram a identificação de escolas, creches, asilos, outras instituições e grupos comunitários, enquanto que o levantamento epidemiológico, que consiste no diagnóstico das doenças bucais mais prevalentes na comunidade, foi encontrado em $43,8 \%$ das equipes (Tabela 3 ).

Sobre a organização do atendimento clínico (Tabela 4), observou-se que as equipes reservam um ou mais turnos da semana para atendimento aos grupos prioritários (82,3\%). A marcação por microárea, por intermédio dos ACS, assim como a agenda aberta, mostraram diferenças significativas, evidenciando serem menos realizadas pelos municípios de grande porte e mais frequentes nos municípios de médio e pequeno porte, respectivamente. Entretanto, o ponto essencial sobre a organização do atendimento está na comparação entre os itens "risco e demanda espontânea”: a marcação por risco é mais encontrada nas equipes dos municípios de grande porte, quando comparada às equipes de pequeno porte $(p=0,001)$, enquanto a marcação por demanda espontânea, método tradicional de marcação das unidades básicas de saúde, mostrou-se mais frequente nos municípios de pequeno porte, quando comparados aos de grande porte.

Com relação à avaliação mensal das ações/ procedimentos realizados pelas ESB (Tabela 4), verificou-se que $76,9 \%$ das equipes desenvolveram essa prática na sua rotina, e $71,4 \%$ dessas utilizaram o mapa de produção, que fornece informações a respeito dos tipos e quantidades de procedimentos realizados pela equipe. Esse instrumento é usado com mais frequência pelas ESB dos municípios de médio e pequeno porte, quando comparados às equipes dos municípios de grande porte $(\mathrm{p}=0,021)$.

A supervisão e a avaliação das ESB pela coordenação de saúde bucal do município demonstraram uma falta de acompanhamento das equi- 
Forma de organização do processo de trabalho das equipes de saúde bucal (ESB) por porte populacional. Pernambuco, Brasil, 2009 (respostas múltiplas).

\begin{tabular}{|c|c|c|c|c|c|c|c|c|c|}
\hline \multirow[t]{2}{*}{ Ações } & \multicolumn{2}{|c|}{ Grande porte } & \multicolumn{2}{|c|}{ Médio porte } & \multicolumn{2}{|c|}{ Pequeno porte } & \multicolumn{3}{|c|}{ Total } \\
\hline & $\mathrm{n}$ & $\%$ & $\mathrm{n}$ & $\%$ & $\mathrm{n}$ & $\%$ & $\mathbf{N}$ & $\%$ & Valor de $p$ \\
\hline \multicolumn{10}{|c|}{ Organização do atendimento clínico } \\
\hline Faixa etária & 35 & 61,4 & 26 & 70,3 & 20 & 74,1 & 83,1 & 68,7 & 0,573 \\
\hline Grupos prioritários & 51 & 89,5 & 29 & 78,4 & 22 & 81,5 & 99,6 & 82,3 & 0,516 \\
\hline Risco & 17 & 29,8 & 3 & 8,1 & 0 & 0,0 & 14,8 & 12,3 & 0,001 \\
\hline Programado & 21 & 36,8 & 6 & 16,2 & 3 & 11,1 & 25,2 & 20,8 & 0,009 \\
\hline Marcação por microárea & 33 & 57,9 & 31 & 83,8 & 21 & 77,8 & 90,7 & 74,9 & 0,025 \\
\hline Agenda aberta & 32 & 56,1 & 27 & 72,9 & 22 & 81,5 & 85,1 & 70,3 & 0,080 \\
\hline Demanda espontânea & 26 & 45,6 & 22 & 59,5 & 21 & 77,8 & 72,7 & 60,1 & 0,053 \\
\hline Outros & 3 & 5,3 & 2 & 5,4 & 1 & 3,7 & 5,9 & 4,9 & 0,886 \\
\hline \multicolumn{10}{|c|}{ A equipe realiza avaliação mensal? } \\
\hline Responderam sim & 35 & 61,4 & 31 & 83,8 & 22 & 81,5 & 93,0 & 76,9 & 0,044 \\
\hline \multicolumn{10}{|c|}{ Que instrumento utiliza para a avaliação? } \\
\hline Relatório do SIA/SUS & 7 & 12,3 & 18 & 48,6 & 7 & 25,9 & 39,6 & 32,7 & 0,001 \\
\hline Mapa de produção & 31 & 54,4 & 29 & 78,4 & 21 & 77,8 & 86,4 & 71,4 & 0,022 \\
\hline \multicolumn{10}{|c|}{ Número de reuniões com a coordenação de saúde } \\
\hline \multicolumn{10}{|c|}{ bucal nos últimos 3 meses } \\
\hline Nenhuma & 21 & 36,8 & 15 & 40,5 & 7 & 25,9 & 43,4 & 35,9 & 0,435 \\
\hline Uma & 10 & 17,5 & 6 & 16,2 & 7 & 25,9 & 22,9 & 18,9 & 0,457 \\
\hline Duas & 8 & 14,0 & 9 & 24,3 & 7 & 25,9 & 26,4 & 21,8 & 0,443 \\
\hline Três & 14 & 24,6 & 7 & 18,9 & 4 & 14,8 & 23,6 & 19,5 & 0,582 \\
\hline Quatro ou mais & 4 & 7,0 & 0 & 0,0 & 1 & 3,7 & 3,5 & 2,9 & 0,245 \\
\hline
\end{tabular}

SIA/SUS: Sistema de Informações Ambulatoriais do Sistema Único de Saúde.

Nota: os valores de N (total), \% (total) e valor de $p$ da tabela acima já se encontram com o fator de ponderação.

pes, visto que $35,9 \%$ delas não participaram de reunião com a coordenação nos últimos 3 meses, e $18,9 \%$ estiveram em apenas uma reunião (Tabela 4).

\section{Discussão}

Neste momento, as práticas realizadas pelas ESB serão comentadas frente ao que preconiza o Ministério da Saúde, baseado na Política Nacional da Atenção Básica ${ }^{6}$, nas Diretrizes da Política Nacional de Saúde Bucal 11 e nos achados de outros autores.

Um fator pouco considerado atualmente como redefinidor das práticas odontológicas na ESF é o enfoque de risco. Por meio desse, é possível identificar as populações com maior risco de desenvolver determinados agravos e, com isso, estabelecer prioridades na atenção à saúde. Segundo alguns autores 12,13, a programação e o planejamento de ações devem estar baseados no diagnóstico das condições de saúde e necessida- des de tratamento da população adscrita, permitindo priorizar grupos mais necessitados e alocar recursos de forma direcionada à modificação positiva das condições de saúde da população, valendo-se de práticas mais efetivas.

A Política Nacional de Atenção Básica 6 refere que a rotina de trabalho das equipes de saúde da família inclui processos de conhecimento do território, da população e também das dinâmicas familiar e social, para o desenvolvimento de ações focalizadas sobre os grupos de risco e fatores de risco comportamentais, alimentares e/ou ambientais, com a finalidade de prevenir o aparecimento ou a manutenção de doenças e danos evitáveis.

As Diretrizes da Política Nacional de Saúde Bucal 11 citam que as ações de promoção da saúde incluem também trabalhar com abordagens sobre os fatores de risco ou de proteção simultâneos, tanto para doenças da cavidade bucal quanto para outros agravos (diabete, hipertensão, obesidade, trauma e câncer), tais como: políticas de alimentação saudável para reduzir o con- 
sumo de açúcares, abordagem comunitária para aumentar o autocuidado com a higiene corporal e bucal, política de eliminação do tabagismo e de redução de acidentes.

Roncalli 14 , em estudo sobre acessibilidade aos serviços odontológicos em Curitiba (Paraná), afirma que, em algumas unidades, de acordo com a realidade local, são estabelecidas formas de priorização baseadas no risco social, sendo as principais estratégias de priorização do ponto de vista mais geral, e que determina o acesso do usuário ao serviço, a idade e a área de abrangência. Esse autor cita que o universo priorizado para Odontologia é composto por: área de risco social, escolas, creches, gestantes, grupos de desnutridos e outros grupos organizados.

No presente estudo, aproximadamente metade das ESB relatou ter realizado o diagnóstico da área adscrita no último ano, o que mostra que muitas das ESB estão planejando as ações sem o conhecimento prévio da realidade local e, consequentemente, realizando atividades que não levam em consideração o critério de equidade. Resultados semelhantes foram encontrados no Rio Grande do Norte, onde o levantamento epidemiológico só foi citado por $43,8 \%$ das equipes. A maioria dos municípios não realiza esses diagnósticos (insatisfatórios) ou, quando os realiza, eles não são instrumentos de trabalho dos dentistas 13 .

A forma de organização do atendimento clínico também reflete a atenção dada aos grupos de risco pelas ESB. O tipo de marcação mais citado foi por meio dos grupos prioritários como gestantes, hipertensos, diabéticos, conforme determina o Ministério da Saúde. Outras formas de atendimento que consideram o risco social e a programação demonstraram ser mais realizadas pelos municípios de grande porte. Entretanto, muitas equipes ainda referiram a marcação por demanda espontânea, principalmente nos municípios de pequeno porte, o que reproduz um modelo tradicional de posto de saúde, em que o paciente somente procura o serviço quando a doença já está instalada e, no caso da Odontologia, quando há dor de dente. Essa forma de atendimento ao paciente é hegemônica historicamente e o fato de estar fortemente relacionada aos municípios de pequeno porte pode ser reflexo da falta de oportunidade de capacitação dos profissionais desses municípios.

Em Alagoinhas (Bahia), a marcação das consultas se dá pela demanda espontânea, usandose fila e distribuição de ficha por ordem de chegada 15. No Estado do Paraná, muitas consultas odontológicas são agendadas nas residências dos usuários ou na própria unidade de saúde da família. Por outro lado, porém, verificou-se que
9,5\% das ESB admitiram que a livre demanda é a porta de entrada para o atendimento odontológico, o que demonstra a dificuldade de adaptação à nova lógica de atenção proposta pelo PSF 9.

Segundo as Diretrizes da Política Nacional de Saúde Bucal 11, o agendamento é um item importante na reordenação da atenção odontológica, que visa a substituir práticas centradas no paradigma cirúrgico-restaurador-mutilador por práticas que considerem o indivíduo como um ser integral, configurando uma evolução no atendimento quando comparado àquele por livre demanda. Entretanto, para que a organização do serviço alcance os resultados desejados é preciso que haja o envolvimento da equipe com a comunidade, informando-a e orientando-a quanto ao ingresso no sistema.

A ESF organiza-se a partir de uma equipe multiprofissional cujo campo disciplinar de atuação é o território-família-comunidade, onde cada um dos profissionais de saúde desenvolve ações de saúde, ora comuns (como as ações de planejamento, busca ativa etc.), ora devendo ser preservadas as suas especificidades. No trabalho em equipe ninguém perde seu núcleo de atuação profissional, devendo-se considerar as diferenças existentes entre as intervenções de cada área 6 .

Com isso, é fundamental a articulação entre as equipes de saúde da família e de saúde bucal, para o desenvolvimento das ações em saúde. Pode-se observar que, em Pernambuco, muitas das ESB participantes referiram ter uma boa integração com a equipe de saúde da família, realizando ações em conjunto como reunião administrativa, planejamento das ações da equipe, participação em campanhas e grupos educativos, dentre outros, sem, entretanto, demonstrarem diferenças estatisticamente significantes entre os municípios. Os dados corroboram com os achados de Baldani et al. 9, os quais constataram que $87,6 \%$ dos dentistas do Paraná relataram haver envolvimento entre a equipe odontológica e o restante da equipe de saúde da família, assim como os achados de Souza \& Roncalli 13, que classificaram como satisfatória a articulação entre a ESB e a equipe de saúde da família na maioria dos municípios estudados no Rio Grande do Norte, por meio da realização de reuniões para avaliação e planejamento das ações das equipes.

Entretanto, pode-se dizer que não é uma tarefa fácil conciliar/integrar as ações dos diferentes profissionais das unidades de saúde da família, pois, segundo as diretrizes citadas anteriormente 11, esses profissionais nem sempre estão preparados e com disposição para agir de forma integrada. O trabalho do CD raramente se insere em práticas partilhadas com profissionais de outras áreas, uma vez que desenvolvem 
suas ações de forma autônoma, independente e individualizada 16, o que vai ao encontro dos resultados desta pesquisa.

Um outro tipo de articulação necessária às ESB ultrapassa as barreiras da própria unidade. É o caso da participação social, estabelecida como característica do processo de trabalho das equipes de Atenção Básica pela Portaria $n^{\circ}$. 648, de 28 de março de 20066 .

Em Pernambuco, observou-se uma razoável integração das ESB com a comunidade, considerando sua participação nos grupos educativos. Entretanto, quando questionadas sobre sua articulação com instituições e outras organizações, verificou-se sua grande participação nas escolas, como vem sendo a participação da Odontologia ao longo do tempo. O maior envolvimento das equipes dos municípios de grande porte com igrejas e creches talvez seja explicado por não haver creches em municípios de pequeno porte e pelo crescente número de igrejas evangélicas nos grandes municípios.

Além disso, a maioria das equipes não tem participantes nas reuniões do Conselho de Saúde, não se reúne com a comunidade para debater sobre cidadania, funcionamento do SUS, assistência prestada, temas de interesse da população, e não existem representantes da comunidade no planejamento de trabalho das ESB.

Crevelim \& Peduzzi 17 relataram, dentre as atividades desenvolvidas pelas equipes da unidade em parceria com voluntários ou outros profissionais, os grupos educativos, teatro nas escolas, dentre outros, o que reproduz o conceito de saúde como qualidade de vida, adotado pelo PSF. Por outro lado, também afirmam que o distanciamento entre equipe e comunidade, ou entre o saber técnico e o saber popular, é evidente em alguns casos, visto que a população da área de abrangência e os usuários estão ausentes das discussões da equipe sobre as necessidades de saúde e o planejamento das ações.

Aerts et al. 18 sugerem as seguintes atividades para o maior envolvimento do cirurgião-dentista com a comunidade:

1. Buscar parceria com ativistas comunitários para a formação de redes e alianças;

2. Apoiar a criação de hortas e pomares como meio de encorajar ações cooperativas e o consumo de alimentos saudáveis;

3. Associar-se com grupos comunitários ativos na promoção da saúde das mães e crianças, como, por exemplo, a pastoral da saúde;

4. Proporcionar a participação da população no planejamento e tomada de decisões em relação à saúde bucal da comunidade;

5. Desenvolver ações intersetoriais com outras instituições públicas ou privadas (ONGs, Serviço
Social da Indústria, Serviço Social do Comércio, universidades).

A avaliação em saúde tem como propósito fundamental dar suporte a todo processo decisório no âmbito do Sistema de Saúde e, por isso, deve subsidiar a identificação de problemas e a reorientação de ações e serviços desenvolvidos, avaliar a incorporação de novas práticas sanitárias na rotina de profissionais e mensurar o impacto das ações implementadas pelos serviços e programas sobre o estado de saúde da população 6 .

Quanto à avaliação das ações de saúde, 76,9\% das ESB referem realizá-la mensalmente, com diferença estatisticamente significante para os municípios de médio e pequeno porte. Há que se considerar a possibilidade desse dado não corresponder à realidade por tratar-se de um questionário autoaplicado. Deve-se observar também que não foi perguntado às equipes a profundidade ou forma de avaliação, podendo ter sido incluídas nessas respostas qualquer tipo de avaliação. Destaque-se que $64,1 \%$ das equipes referem fazer reunião periódica com a coordenação de saúde bucal, na qual pode haver avaliações, e que $71,4 \%$ das ESB referem usar o mapa de produção como instrumento de avaliação.

Em muitos municípios brasileiros, a ausência da rotina de monitoramento e avaliação das ações de saúde é um problema sério a ser enfrentado pelos gestores. Leal \& Tomita 19 observaram, em seus estudos realizados em dois municípios do Estado de São Paulo, a falta de mecanismos de acompanhamento, controle e avaliação das ações realizadas pelos serviços odontológicos municipais.

Na tentativa de avançar nessa questão, é interessante que os profissionais de saúde, gerentes de unidades ou profissionais das equipes de saúde da família, utilizem os sistemas de informação disponíveis, dentre eles o Sistema de Informação da Atenção Básica (SIAB) e o Sistema de Informações Ambulatoriais do SUS (SIA/SUS), como uma maneira mais ágil e prática de conseguir os dados necessários, pois os processos avaliativos devem ser incorporados às práticas dos serviços de saúde não só para o planejamento, mas para a tomada de decisão 20 .

É interessante também ressaltar a importância do acompanhamento das equipes pelo coordenador de saúde bucal, que tem a responsabilidade de organizar o modelo de atenção à saúde bucal do município e orientar as equipes quanto às suas responsabilidades e ao cumprimento dos princípios e diretrizes do SUS na ESF. Em alguns municípios de Pernambuco, há a necessidade de uma maior participação dos coordenadores, considerando que muitas equipes não se 
reuniram com a coordenação de saúde bucal no período de 3 meses.

A abordagem multiprofissional, a participação social, o diagnóstico da saúde da comunidade, o planejamento, o monitoramento e a avaliação das ações são questões apresentadas como norteadoras para o sucesso da ESF 6. Para Paim 21, as tecnologias desenvolvidas, as formas como a demanda devem ser organizadas, as estratégias de inclusão, o incentivo à participação popular etc. são desdobramentos importantes na consolidação do SUS e cada nível deve pensar a melhor maneira de colocá-los em prática.

Podem existir modelos que desenvolvam exclusivamente intervenções de natureza médicocurativa e outros que incorporem ações de promoção e prevenção, e ainda há modelos em que seus serviços simplesmente atendem às demandas ou que atuem ativamente sobre os usuários. Outros modelos (ou propostas de modelos) podem ser resgatados na literatura em saúde coletiva e todos são, na verdade, tentativas (algumas bem, outras malsucedidas) de implementar o SUS em nível municipal 21.

Martelli et al. 22 corroboram com essa afirmativa, pois em estudo de revisão em que foram analisados 21 artigos científicos, não houve nenhum caso de modelo que se proclamasse antagônico ao ideário SUS ou que relatasse experiências não compatíveis com o mesmo.

Sobre esses aspectos, com relação às ESB do Estado de Pernambuco, pode-se dizer que ainda existe uma dificuldade das equipes organizarem seu processo de trabalho, embora apresente características do modelo de vigilância da saúde, com práticas que envolvem intervenções sobre os problemas de saúde, ênfase em problemas que requerem acompanhamento contínuo, articulação entre ações promocionais, preventivas e curativas, além de ações sobre o território.

Um dos fatores limitantes deste estudo refere-se ao seu desenho do tipo transversal, que limita a interpretação dos resultados, não sendo possível estabelecer relações entre causa e efeito. Outra limitação deste estudo está no fato de que as informações utilizadas para se avaliar a atuação das equipes eram autorreferidas, não sendo possível, assim, comprovar se as ações citadas pelos profissionais eram realmente executadas. Espera-se que este artigo sirva de base para estudos futuros que possam investigar em profundidade alguns aspectos desse trabalho ou complementar com informações que foram pouco exploradas.

\section{Conclusão}

No processo de organização do trabalho das ESB, apesar da integração observada com os demais membros da equipe de saúde da família e a realização de avaliações mensais, outras ações como o diagnóstico da situação de saúde e utilização do mapa de área, são práticas pouco realizadas e que dificultam a efetividade das ações de saúde. A forma de organização do atendimento clínico evidenciou a atenção aos grupos prioritários, embora a agenda aberta e a demanda espontânea ainda sejam uma realidade em muitos municípios.

A articulação com a comunidade também necessita de avanços. Embora observada sua participação nos grupos educativos, em especial de gestantes, idosos e hipertensos/diabéticos, a integração com instituições da comunidade ainda está muito focada nas escolas, privando as equipes de outras formas de participação comunitária.

Considerando o porte populacional dos municípios, pode-se observar que grande parte das variáveis estudadas não apresentou diferença estatisticamente significante, entretanto foi possível constatar: a integração com instituições comunitárias como igrejas e creches está associada às equipes dos municípios de grande porte, além do atendimento clínico por risco social e pela programação. A marcação por microárea, por intermédio dos ACS, assim como a agenda aberta e a demanda espontânea, mostraram ser mais frequentes nos municípios de médio e pequeno porte.

Dessa forma, a incorporação dos pressupostos da ESF poderá ser obtida por meio do estímulo à educação permanente, almejando reduzir muitas das diferenças encontradas entre os municípios de pequeno, médio e grande porte do Estado de Pernambuco. 


\section{Resumo}

O estudo teve como objetivo analisar o processo de trabalho das equipes de saúde bucal no Estado de Pernambuco, Brasil, segundo porte populacional, enfocando a articulação junto à comunidade e a organização do atendimento clínico. Foram aplicados questionários estruturados a 121 equipes de saúde bucal de 29 municípios. Para verificar a associação entre as ações realizadas e o porte populacional dos municípios, utilizou-se o teste qui-quadrado. Na integração das equipes com a comunidade, observou-se o maior foco nas escolas $(81,2 \%)$, entretanto a integração com igrejas ( $p$ $=0,000)$ e creches $(p=0,000)$ demonstrou associação com os municípios de grande porte. A forma de organização do atendimento clínico evidenciou a atenção aos grupos prioritários (82,3\%), sendo ainda bastante presente a marcação por demanda espontânea, principalmente nos municípios de pequeno porte. Assim, é necessário investir em educação permanente para a adequação dos processos de trabalho realizados pelas equipes e a redução das diferenças entre as práticas realizadas nos diversos municípios de Pernambuco.

Odontologia em Saúde Pública; Serviços de Saúde Bucal; Programa Saúde da Família; Saúde Bucal

\section{Colaboradores}

F. C. Pimentel e P. C. Albuquerque participaram da elaboração do estudo, análise dos dados, redação e revisão final do artigo. W. V. Souza participou da elaboração do estudo, análise e interpretação dos dados. P. J. L. Martelli e R. M. L. Acioli participaram da coleta dos dados, discussão dos resultados e redação do manuscrito.

\section{Referências}

1. Zanetti CHG. Por um caminho sustentável para universalização da atenção básica. http://www. saudebucalcoletiva.unb.br/oficina/estado/cole tivo_amplo/psf_caminho.htm (acessado em 04/ Ago/2007).

2. Oliveira JLC, Saliba NA. Atenção odontológica no Programa de Saúde da Família de Campos dos Goytacazes. Ciênc Saúde Coletiva 2005; 10:297-302.

3. Silva SF. A saúde bucal dentro da estratégia de saúde da família no Estado de Pernambuco. [Monografia de Residência]. Recife: Centro de Pesquisas Aggeu Magalhães, Fundação Oswaldo Cruz; 2006.

4. Viana IB. Análise da evolução das Equipes de Saúde Bucal na Estratégia de Saúde da Família em Pernambuco no período de 2001 a 2009. [Monografia de Residência]. Recife: Centro de Pesquisas Aggeu Magalhães, Fundação Oswaldo Cruz; 2011.
5. Giovanella L, Escorel S, Mendonça MHM. Porta de entrada pela atenção básica? Integração do PSF à rede de serviços de saúde. Saúde Debate 2003; 27:278-89.

6. Brasil. Portaria ${ }^{\circ}$. 648 de 28 de março de 2006. Diário Oficial da República Federativa do Brasil 2006; 29 mar.

7. Araújo MBS, Rocha PM. Trabalho em equipe: um desafio para a consolidação da estratégia de saúde da família. Ciênc Saúde Coletiva 2007; 12:455-64.

8. Silveira Filho AD. A Saúde Bucal no PSF: o desafio de mudar a prática. http://paginas.terra.com.br/ saude/angelonline/artigos/art_psf/artigo_rbsf_ toni.pdf (acessado em 12/Jan/2009). 
9. Baldani MH, Fadel CB, Possamai T, Queiroz MGS. A inclusão da odontologia no Programa de Saúde da Família no Estado do Paraná, Brasil. Cad Saúde Pública 2005; 21:1026-35.

10. Abrahão AL, Freitas CSF. Modos de cuidar em saúde pública: o trabalho grupal na rede básica de saúde. Rev Enferm UERJ 2009; 17:436-41.

11. Coordenação Nacional de Saúde Bucal, Departamento de Atenção Básica, Secretaria de Atenção à Saúde, Ministério da Saúde. Diretrizes da Política Nacional de Saúde Bucal. http://bvsms.saude.gov. br/bvs/publicacoes/politica_nacional_brasil_sor ridente.pdf (acessado em 12/Jan/2009).

12. Mota E, Carvalho D. Sistemas de informação em saúde. In: Rouquayrol MZ, Almeida Filho N, organizadores. Epidemiologia \& saúde. 6a Ed. Rio de Janeiro: Medsi; 1999. p. 505-21.

13. Souza TMS, Roncalli AG. Saúde bucal no Programa Saúde da Família: uma avaliação do modelo assistencial. Cad Saúde Pública 2007; 23:2727-39.

14. Roncalli AG. A organização da demanda em serviços públicos de saúde bucal: universalidade, eqüidade e integralidade em Saúde Bucal Coletiva. 2000 [Tese de Doutorado]. Araçatuba: Faculdade de Odontologia de Araçatuba, Universidade Estadual Paulista Júlio de Mesquita Filho; 2000.

15. Rodrigues AAAO, Assis MMA. Oferta e demanda na atenção à saúde bucal: o processo de trabalho no Programa Saúde da Família em Alagoinhas-Bahia. Rev Baiana Saúde Pública 2005; 29:273-85.
16. Teixeira MCD. A dimensão cuidadora do trabalho de equipe em saúde e sua contribuição para a odontologia. Ciênc Saúde Coletiva 2006; 11:45-51.

17. Crevelim MA, Peduzzi M. Participação da comunidade na equipe de saúde da família: é possível estabelecer um projeto comum entre trabalhadores e usuários? Ciênc Saúde Coletiva 2005; 10:323-31.

18. Aerts D, Abegg C, Cesa K. O papel do cirurgiãodentista no Sistema Único de Saúde. Ciênc Saúde Coletiva 2004; 9:131-8.

19. Leal RB, Tomita NE. Assistência odontológica e universalização: percepção de gestores municipais. Ciênc Saúde Coletiva 2006; 11:155-60.

20. Pimentel FC, Martelli PJL, Araújo Junior JLAC, Lima AS, Santana VGD, Macedo CLSV. Evolução da assistência em saúde bucal na Estratégia de Saúde da Família do Município do Recife (PE) no período de 2001 a 2007. Rev Baiana Saúde Pública 2008; 32:253-64.

21. Paim J. Reforma sanitária e os modelos assistências. In: Rouquayrol MZ, Almeida Filho N, organizadores. Epidemiologia \& saúde. 5a Ed. Rio de Janeiro: Medsi; 1999. p. 473-88.

22. Martelli PJL, Araújo Júnior JLAC, Pimentel FC. Modelos municipais em saúde bucal: tendências atuais. Int J Dent 2009; 8:146-59.

Recebido em 02/Jan/2011

Versão final reapresentada em 11/Jul/2011

Aprovado em 19/Jul/2011 